\title{
Patterns to Guide Practical Refactoring: examples targetting promotion in $\mathrm{Z}$
}

\author{
Susan Stepney, Fiona Polack, and Ian Toyn \\ Department of Computer Science, University of York, \\ Heslington, York, YO10 5DD, UK. \\ \{susan,fiona,ian\}@cs.york.ac.uk
}

\begin{abstract}
Formal methods such as $\mathrm{Z}$ are generally criticised for their lack of practical applicability. As in other areas of software engineering, patterns help to construct, analyse and describe formal texts. Once a method has a catalogue of patterns, development can proceed by applying patterns, and by moving from one sort of pattern to another. This paper illustrates a developmental use of patterns. First, we describe the set of patterns that collectively represent the well-known $\mathrm{Z}$ structure, promotion. We then show how refactoring can be used to take an unstructured $\mathrm{Z}$ specification in to a promotion structure.
\end{abstract}

Keyword: Z, patterns, refactoring, development methods

\section{Introduction}

Pattern languages [Alexander et al. 1977] [Gamma et al. 1995] and refactoring [Fowler 1999] are programming techniques that can also be fruitfully applied to specification [Stepney et al. 2003a] [Stepney et al. 2002].

In this paper, we illustrate how patterns and refactoring can be applied, using the example of $\mathrm{Z}$ promotion as an elaboration of the Delta/Xi pattern [Stepney et al. 2003a]. We describe the simplest case of the promotion pattern in terms of a set of sub-patterns that can be used as steps in generating a promoted specification. We then provide various elaboration patterns, showing how the simple case can be adapted to different circumstances. Finally we use the promotion generative sub-patterns as a basis for refactoring, to take an unstructured $\mathrm{Z}$ specification into a promotion structure.

\section{The Promotion pattern, and its generative sub-patterns}

\footnotetext{
Promotion

Intent: Specify a global system in terms of multiple instances of a local state, and of operations that manipulate a local state.

Problem: A system that is essentially a hierarchy of components has operations and state at different levels in the hierarchy. This is difficult to specify directly, requiring $\mathrm{Z}$ structures such as $\mu$ and $\theta$ schema bindings.
} 
Solution: Build a specification for local state and operations. Build the global state as a composite of local states. Define framing schemas to promote the local operations. Use schema calculus to construct appropriate global operations. (These steps are expanded further in the four sub-patterns below.)

Specimens: Unix File System [Morgan \& Sufrin 1984] (first published example); [Woodcock \& Davies 1996] (useful refinement laws); [Stepney \& Cooper 2000] (an operating system for managing processes on a smart card).

We structure the promotion pattern into four sub-patterns, used to guide the generation of a promoted specification. For reasons of space, we merely summarise these sub-patterns here, giving the intent and the solution only. See [Barden et al. 1994, chapter 19] for a fuller description of the underlying structure. [Stepney et al. 2003b] gives an example of using these sub-patterns to generate a promoted specification, starting from a local state specification.

The sub-pattern illustrations are documented according to the presentation pattern comment the intent, and illustrated by diagramming the structure [Stepney et al. 2003a].

Promotion: local state and operations

Intent: describe the state, operations, initialisation and (precondition for) finalisation, for a single instance of the multi-instance system.

Solution:

$$
\begin{aligned}
& \text { Local }==[\text { state components } \mid \text { constraints }] \\
& \text { LocalOp }==[\Delta \text { Local; a? : IN; b! : OUT } \mid \text { constraints }] \\
& \text { LocalInit }==\left[\text { Local }^{\prime} \mid \text { constraints }\right] \\
& \text { LocalFinalise }==[\text { Local } \mid \text { constraints }]
\end{aligned}
$$

\section{Promotion: global state}

Intent: describe the global context of a multi-instance system.

Solution:

- the global state requires a set, $[I D]$ of identities

- gbl maps identities to instances of the local state

$$
\text { Global }==[g b l: I D \rightarrow \text { Local }]
$$

\section{Promotion: framing schemas}

Intent: provide a context that describes how the global state is updated by the results of local operations. 
Solution: the context, or "frame" is written as a "framing schema". This establishes the relationship of the state of a local instance to the global state, then shows how the after-state of an operation on that local instance is used to update the global state (the after-state of any suitable global operation) - no details of the global operation or the local operation are required; the frame merely deals with states established by operations defined elsewhere.

The following frame is for any operation that updates the state(s).

- $\Delta$ Global, $\Delta$ Local introduce before and after global and local states

$-x$ ? is the global identity of an (existing) local instance

\begin{tabular}{l} 
ФUpdate \\
$\Delta$ Global \\
$\Delta$ Local \\
$x ?: I D$ \\
\hline$x ? \in \operatorname{dom} g b l$ \\
$\theta$ Local $=g b l x ?$ \\
$g b l^{\prime}=g b l \oplus\left\{x ? \mapsto \theta\right.$ Local $\left.^{\prime}\right\}$ \\
\hline
\end{tabular}

$-x ?$ is in the global set of local instances

- the before state of Local is the current local state associated with $x$ ?

- the global state is updated by overriding the $g b l$ function with the maplet from $x$ ? to the after-state of Local

The frame for introducing a new local instance to the global state is similar to an initialisation.

- declarations are as above, except that there is no Local before state

\begin{tabular}{|l|} 
ФNew \\
$\Delta$ Global \\
Local' \\
$x ?: I D$ \\
\hline$x ? \notin \operatorname{dom} g b l$ \\
$g b l^{\prime}=g b l \cup\left\{x ? \mapsto \theta\right.$ Local $\left.^{\prime}\right\}$ \\
\hline
\end{tabular}

$-x$ ? does not have a mapping in the global state (it is an unused identity)

- The $g b l$ function is updated by set union, adding the mapping from $x$ ? to the after-state of Local

The frame for an operation to remove a local instance from the global state, ФRemove, is similar, but removes the local instance from the global mapping. The frame is needed only where the removal of a local instance must be verified using operations at the local level. 

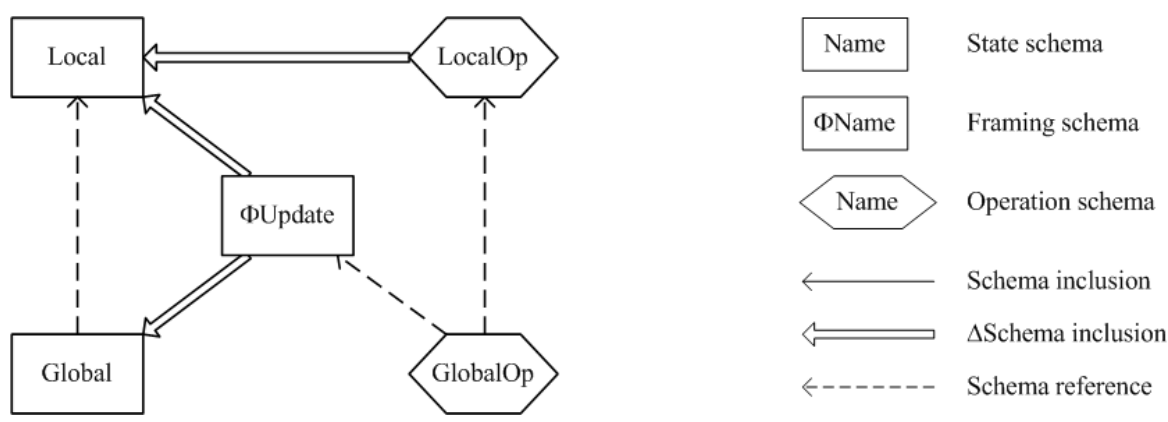

Fig. 1. Structure of the promotion pattern.

- declarations are as above, except that there is no Local after state

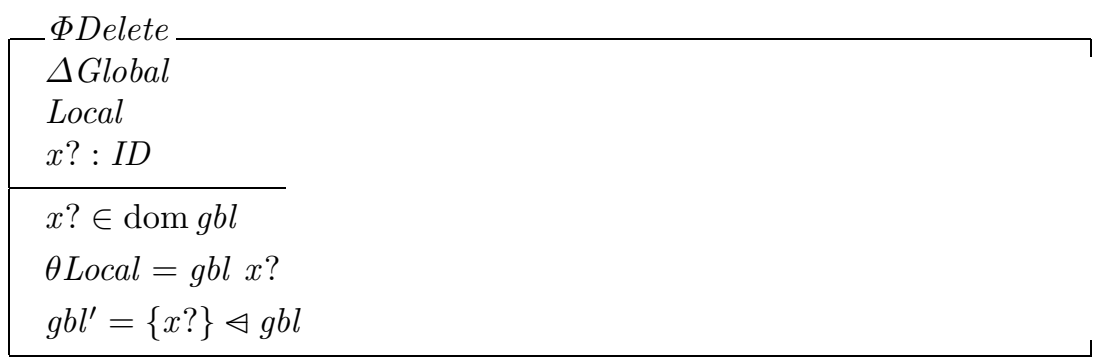

$-x ?$ is in the global set of local instances

- the before-state of Local is the current local state associated with $x$ ?

- the global state is updated by removing the relevant maplet from $g b l$

\section{Promotion: global operations}

Intent: write a global operation in terms of the defined local operations.

Solution: Use schema calculus to extract the relevant local operation (or afterstate of a local operation) and conjoin the appropriate framing schema.

$$
\begin{aligned}
& \text { GlobalOp }==\exists \Delta \text { Local } \bullet \Phi \text { Update } \wedge \text { LocalOp } \\
& \text { GlobalNew }==\exists \text { Local }^{\prime} \bullet \Phi \text { New } \wedge \text { LocalInit } \\
& \text { GlobalRemove }==\exists \text { Local } \bullet \Phi \text { Remove } \wedge \text { LocalFinalise }
\end{aligned}
$$

Define an appropriate global initialisation:

$$
\text { GlobalInit }==[\text { Global' } \mid \text { constraints }]
$$

Note that the type of GlobalOp is [ $\triangle$ Global; $x$ ? :ID; $a ?: I N ; b$ ! : OUT ].

A diagrammatic representation of the promoted update operations is shown in figure 1. (For a fuller description of the diagrammatic notation, based on an extension of that in [d'Inverno \& Luck 2001], see [Stepney et al. 2003a].) 


\section{Elaboration Patterns for Promotion}

We now present various elaborations of the promotion pattern, for coping with cases that do not fit the simple pattern.

\section{Promotion (elaboration): global constraints}

Intent: add global state components to the collection of local instances Solution:

GlobalWithState

\section{Global}

purely global state components

constraints on global state

constraints relating local states

The addition of global state components may require addition of constraints (a) on the global state, and (b) relating the local instance states; the promoted local operations may affect the global state through such constraints. There may also be operations that act on the global state components alone.

Illustration: [Stepney et al. 2000] defines a collection of concrete (that is, refined from the abstract) electronic purses. The global state has additional mechanisms to control interoperability of the purses. The extended global state is:

- conPurse is the global state for the local purses, ConPurse, using identities from the set, NAME

- ether and archive are global-only state elements

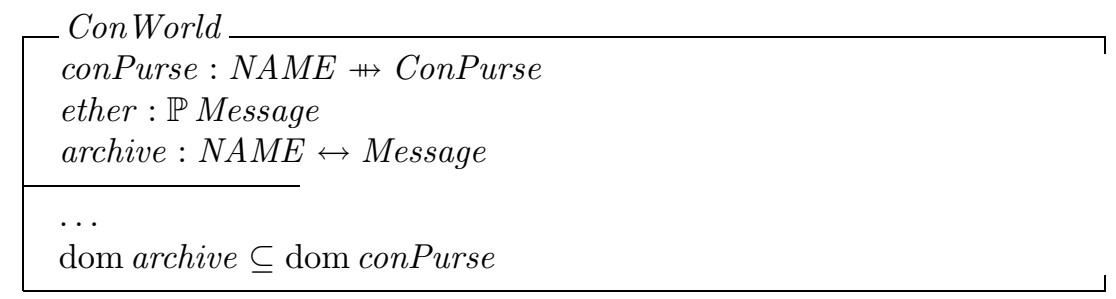

- the global state element, archive, is constrained to only known local purses

All the single purse operations are promoted to the global level. The inputs and outputs from the local operation, $m$ ? and $m$ !, are not simply mapped into global inputs and outputs; they are also linked to the ether (the message transport mechanism) by the framing schema, which extends the framing schemas pattern: 


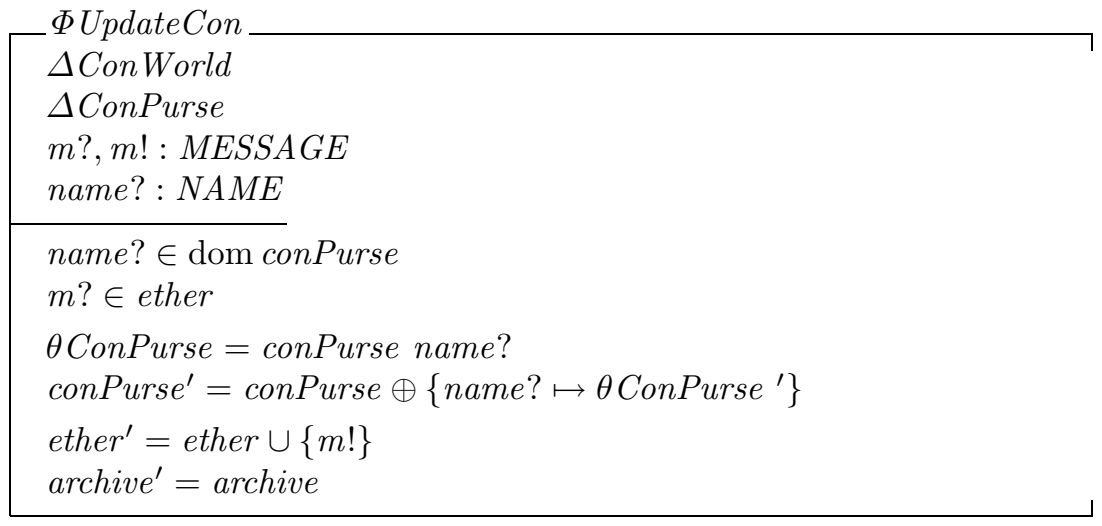

In addition to the promoted operations, there are some global-only operations, for example to add messages in the ether to the archive

\section{Promotion (elaboration): internal identifiers}

Intent: use a native element of the local state as the identifier. (The global identifiers used in promotion are arbitrary. Where the local state has its own identity, this may be a suitable global identifier.)

Solutions:

There are two solutions, depending on whether the promotion uses only internal identity, or uses both internal and global identity redundantly.

Solution 1: Internal identity only

Adapt Promotion: local state and operations thus:

Include the identity as a native attribute of the local state

$$
\text { Local }==[\text { self }: I D ; \ldots]
$$

Include a constraint in local operations that the identity is not changed by the operation

$$
\text { LocalOp }==\left[\Delta \text { Local; } \ldots \mid \text { self }{ }^{\prime}=\text { self } \wedge \ldots\right]
$$

Adapt Promotion: global state thus:

Make the global state the set of local instances, with different local states having different identities.

$$
\text { Global }==[\text { gbl }: \mathbb{P} \text { Local } \mid \forall x, y: \text { glb } \mid x \neq y \bullet x . s e l f \neq y . \text { self }]
$$

Global operations must include constraints to check the identity of local instances. For exapmle, the frame for the update operations modifies the predicates of the framing schemas pattern as follows. 


\begin{tabular}{|l|}
\hline SUpdate \\
$\Delta$ Global \\
$\Delta$ Local \\
$x ?: I D$ \\
\hline self $=x ?$ \\
$\theta$ Local $\in$ gbl \\
gbl' $=($ gbl $\backslash\{\theta$ Local $\}) \cup\left\{\theta\right.$ Local $\left.^{\prime}\right\}$ \\
\hline
\end{tabular}

- the local state's self component matches the given identifier, $x$ ?

- the local instance occurs in the global state $g b l$

- the update replaces the local instance in the global set of instances with the result of the local operation

The frame for the operation to add a new piece of local state is as follows.

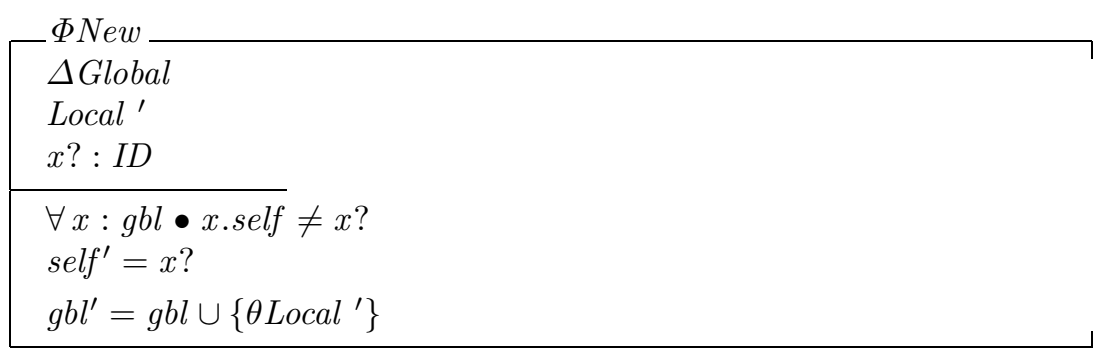

- no pre-existing local state has the given identifier, $x$ ?

- the new local state has identifier $x$ ?

- the update adds the new local state to the global set

Solution 2: External and internal identities

The local state and operation definitions are the same as for solution 1 .

Adapt Promotion: global state thus:

Make the global function an injection, capturing the one-to-one correspondence between 'external' and 'internal' names, and ensure that, for any instance, external and internal identities are the same.

$$
\text { Global }==[g b l: I D \mapsto \text { Local } \mid \forall x: \operatorname{dom} g b l \bullet(g b l x) . s e l f=x]
$$

Example: In the concrete specification of the electronic purse, the purse has a name; this is the name that the purse is known by at the external level. The local state is ConPurse:

$$
\text { ConPurse }==[\text { name }: \text { NAME } ; \ldots \mid \ldots]
$$

Adapt Promotion: global state thus:

- ConWorld uses the same type as the local identifier for the domain of the global conPurse. 


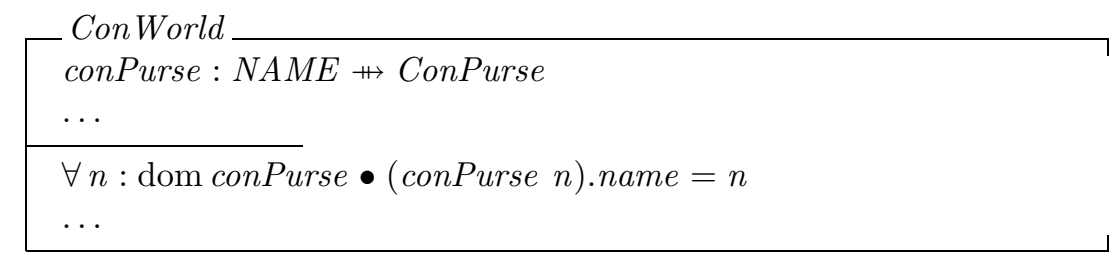

- every purse's internal name must be the same as the name by which it is known in the global system.

The framing schemas pattern is applicable, as the additional constraint is carried forward in the $\Delta$ Con World inclusion.

Specimen: Hall's style [Stepney et al. 1992, chapter 3] combines both these variants. It has a set of local states, and it has a derived mapping from (external) identities to local states:

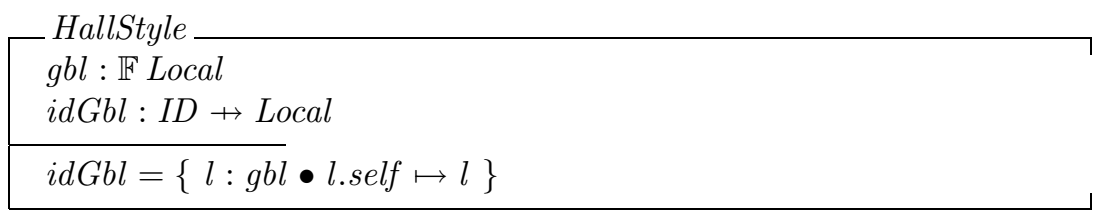

\section{Promotion (elaboration): combine promotions}

Intent: specify a system that conforms to the local-global format for the promotion pattern, but has different kinds of local instance.

Solution:

- Each kind of local instance is associated with a separate component of the global state.

$$
\text { Global }==[\text { gbl1:ID1 } \rightarrow \text { Local } 1 ; \ldots ; \text { gbln }: I D N \rightarrow \text { LocalN }]
$$

- Constraints could be added as appropriate.

Example: [Stepney \& Cooper 2000]'s specification of a Smart Card Operating System specifies three types of application instance that the operating system has to control: fixed ISO applications, user programmable applications, and (for modelling reasons) 'absent' applications. It is possible to promote these three types of local instances individually:

- fixed and user follow the global state pattern.

- absent has no local state other than an identifier, so the internal identifier pattern of promotion is applied. 
CardGlobal

fixed $: I D \rightarrow$ Fixed

user $: I D \rightarrow A p p l$

absent $: \mathbb{P} I D$

disjoint $\langle$ dom fixed, dom user, absent $\rangle$

- In this case, the global identifiers for each promotion are drawn from the same set, ID, and so are constrained to be disjoint.

Alternatively, combine the various local types with a free type disjoint union, and use this in the simple promotion pattern. (This results in much extracting of state from the free type machinery, however.)

\section{Promotion (elaboration): multi-promotion}

Intent: specify a system that comprises multiple instances, but has global operations that may affect more than one local instance.

Solutions: the local state and operations and global state patterns are applied. The framing schemas include multiple local instances. There are two possible patterns. The first applies when the global operations affect a fixed number of local instances. The second is more general, and applies when the number of local operations affected by the global operation is variable or unknown.

Solution 1: A fixed number of local instances, illustrated for two instances, but extensible to as many as are practical or desired.

Adapt Promotion: framing schemas thus:

- The (otherwise identical) local states are declared twice, distinguished by decorations. (Note that in $\Delta$ Local $_{1}$, the decoration applies to the whole $\Delta$ Local phrase, and thus yields Local $_{1}$ and Local $^{\prime}{ }_{1}$.)

\begin{tabular}{l} 
ФUpdate 2 \\
$\Delta$ Global \\
$\Delta$ Local $_{1}$ \\
$\Delta$ Local $_{2}$ \\
$x ?, y ?: I D$ \\
\hline$\{x ?, y ?\} \subseteq \operatorname{dom} g b l$ \\
$x ? \neq y ?$ \\
$\theta$ Local $_{1}=g b l x ?$ \\
$\theta$ Local $_{2}=g b l y ?$ \\
gbl' $=g b l \oplus\left\{x ? \mapsto \theta\right.$ Local $^{\prime}{ }_{1}, y ? \mapsto \theta$ Local $\left._{2}\right\}$
\end{tabular}

- the provided identifiers are different instances from the set of identifiers used in the global system 
- each local state instance is bound to one of the input identifiers

- the result of the global operation overrides the global state with the mappings from each identifier to the after-state of the local operation on its local state

Then the global operation becomes

$$
\begin{aligned}
\text { GlobalOp }== & \exists \Delta \text { Local }_{1} ; \Delta \text { Local }_{2} \bullet \\
\text { SUpdate } 2 & \wedge \text { ALocalOp }_{1} \wedge \text { AnotherLocalOp }_{2}
\end{aligned}
$$

The type of GlobalOp is [ $\Delta$ Global; $x$ ? :ID; $a ?_{1}, a ?_{2}: I N ; b !_{1}, b !_{2}:$ OUT ]. If the inputs or output need to be the same for both operations, renaming is required.

Illustration: This pattern is illustrated in the specification of the electronic purse transfer operations, section 4.5.

Solution 2: An arbitrary number of local instances

If there is an arbitrary number of local states all affected by the same operation, they can be bundled into a sequence $l s$, with a corresponding sequence of inputs, $x s$ ?, which identifies which local instances are being promoted. The framing schema becomes

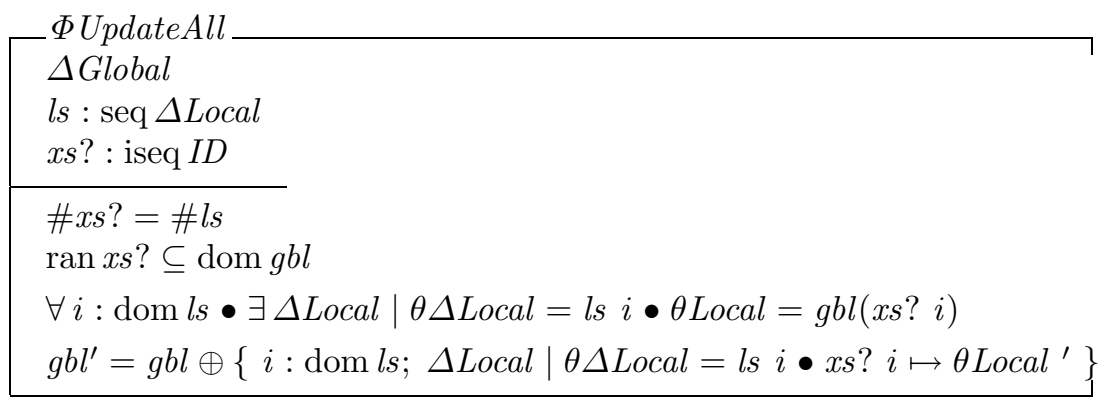

The local operation is cast into a form for use with the framing schema:

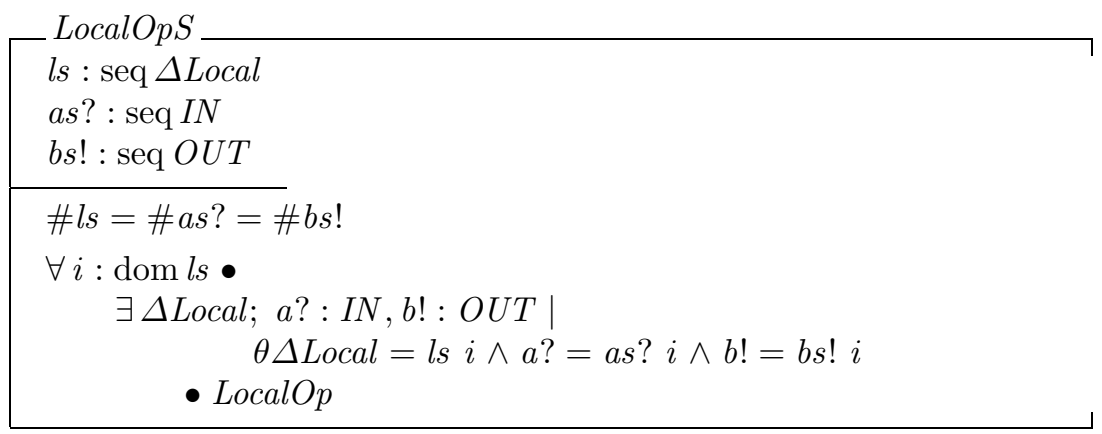

Then the global operation becomes

GlobalOp $==\exists l s:$ seq $\Delta$ Local $\bullet \Phi$ UpdateAll $\wedge$ LocalOpS 
The type of GlobalOp is [ $\triangle$ Global; xs? : iseq ID; as? : seq IN; bs! : seq OUT ]. Illustration: Consider a local state, Counter that holds an incrementable natural number. An operation on the local state resets the number to zero. Following the local state and operations pattern, these are defined:

$$
\begin{aligned}
& \text { Counter }==[c: \mathbb{N}] \\
& \text { Reset }==\left[\Delta \text { Counter } \mid c^{\prime}=0\right]
\end{aligned}
$$

A global system of counters can be defined using the global state pattern:

$$
\text { CounterSystem }==[\text { sys }: N A M E \rightarrow \text { Counter }]
$$

To simultaneously reset all the identified counters to zero (assuming a $\Phi$ UpdateAll defined following the pattern above, but using the names of this example):

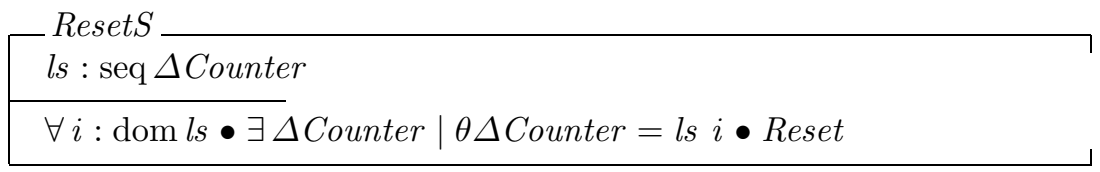

$$
\text { ResetAll }==\exists l s: \text { seq } \Delta \text { Counter } \bullet \Phi U p d a t e A l l \wedge \text { Reset } S
$$

This is equivalent to

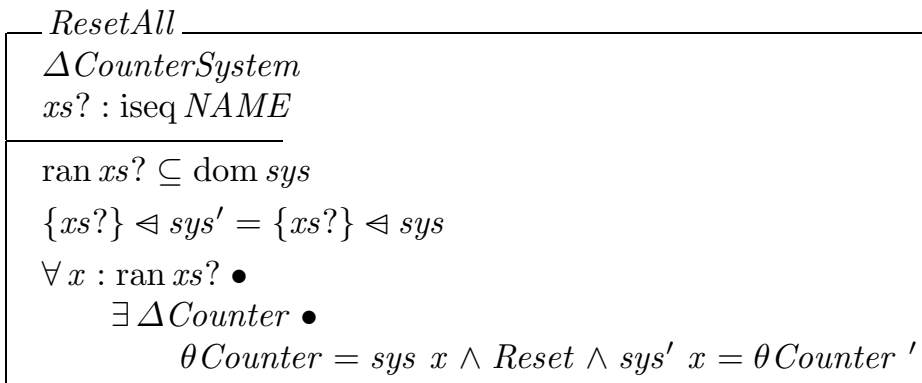

\section{Using the promotion patterns}

The promotion pattern and its sub-patterns can be used to generate a specification with a promotion structure. For example, [Stepney et al. 2003b] show how to use the patterns to generate a promoted bank account specification from a single account specification.

Here we focus on using the promotion patterns to generate a refactoring of an existing unstructured specification. We start with an initial specification 
closely based on the 'Abstract World' specification from the electronic purse development [Stepney et al. 2000].

The structural promotion pattern is the target pattern for the refactoring, and the generative patterns guide the refactoring steps. Some of the refactor transformations do not seem obvious and might be missed without the generative patterns as a reference. Indeed, some of the transformations temporarily make the specification structure more baroque.

\subsection{Starting point: the existing specification}

- purses are identified by a name

$[N A M E]$

The abstract world comprises two mappings

- balance maps purse names to their balances

- lost maps purse names to the total amount they have lost because of failed transfer operations

- modelling notes: We wish to distinguish empty purses from non-existent purses, so we use a partial function rather than a bag. We use a finite function to ensure that the total balance in the system is well-defined.

World $==[$ balance, lost $: N A M E \pitchfork \mathbb{N} \mid$ dom balance $=$ dom lost $]$

- the balance and lost domains are the same, and define the purse names known to the system

There is a standard precondition for operations involving transfer between two purses. This is specified separately and included in each operation to aid readability (following the name predicates pattern [Stepney et al. 2003b]).

- transfer operations always have two input purse names and an input representing the value to be transferred between the purses

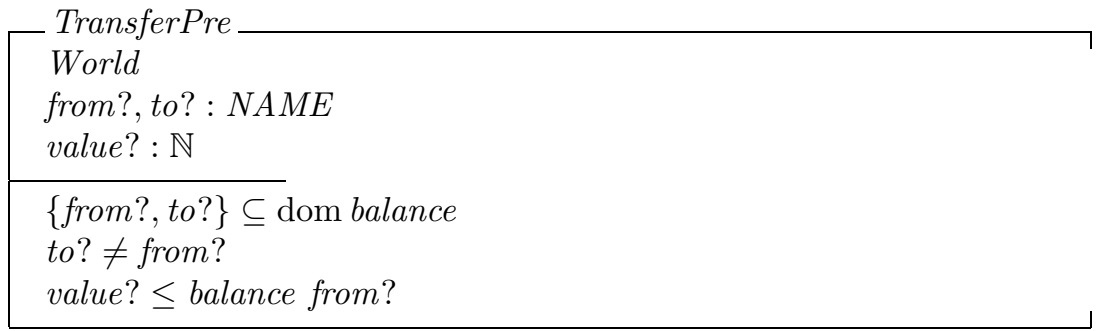

- the two identified purses are known and distinct (it does not make sense to transfer money from one purse to itself) 


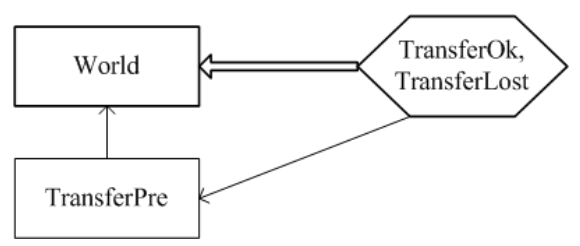

Fig. 2. Structure of the abstract world of electronic purses, before refactoring

- there is sufficient funds for the transfer

There are two transfer operations; one that succeeds, and one that fails. Both have the same declarations.

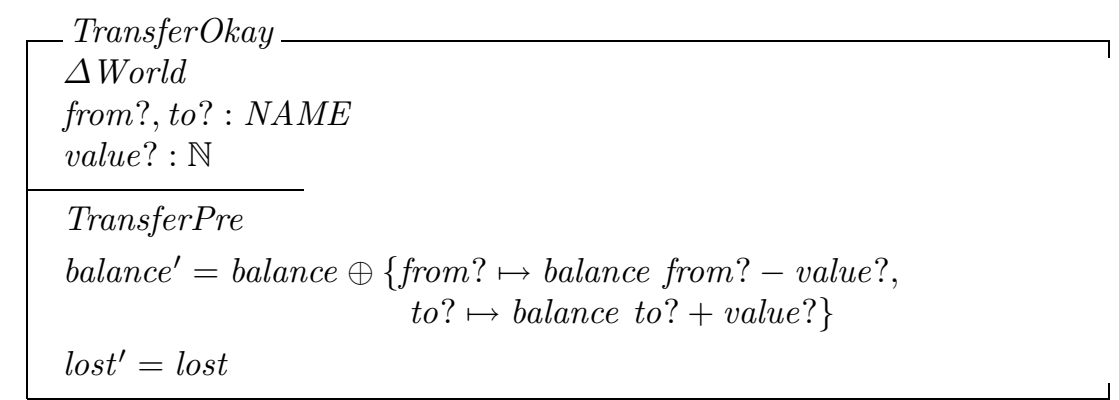

- a successful transfer decrements value? from the from? purse's balance, and adds it to the to? purse's balance, leaving all other balances unchanged

- success means the lost components are unchanged

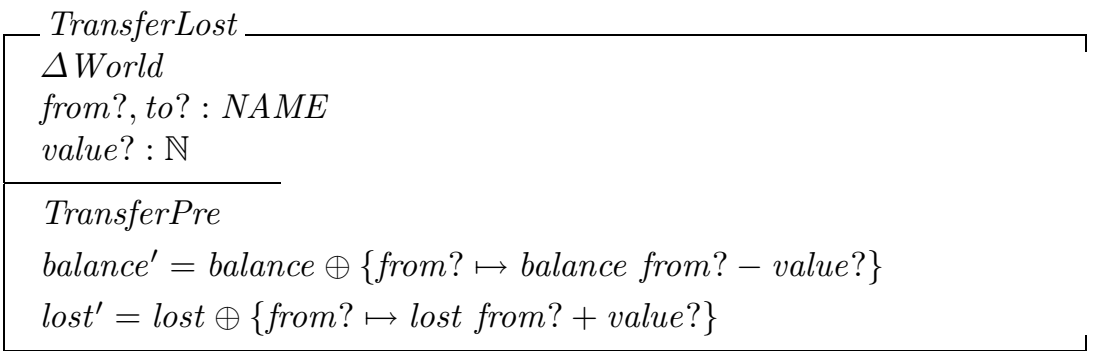

- a lost transfer decrements value? from the from? purse's balance, and loses it, modelled by adding it to the from? purse's lost component

- no other purse is affected

Figure 2 represents the structure of the specification before refactoring. It is a simple Delta/Xi pattern; there is also a schema included (as a precondition) in the operations.

The purse system is a Delta/Xi specification that matches the multi-promotion intent: it describes a system made up of instances of a local state and global operations based on operations on multiple (two) local states. 
The refactoring steps are based on the four elements of the generative pattern for promotion. The first generative pattern, local state and operations, requires substantial refactoring over several steps. The second generative pattern, global state, emerges as a side effect.

\subsection{Step 1: introduce local state}

The first goal for refactoring is to define the local state. The system described above has a collection of purses, so the purse becomes the local instance.

The individual purses each have a balance and a lost component. The schema is formed by removing the identifying functions and predicates from the original state specification. The result matches the state of local state and operations:

$$
\text { Purse }==[\text { balance, lost }: \mathbb{N}]
$$

Global elements of the original state are refactored to match global state:

$[N A M E]$

$$
\text { World }==[\text { purse }: \text { NAME } \pitchfork \text { Purse }]
$$

The preservation of meaning for purse can be established by a schema expansion refactoring [Stepney et al. 2002] to a single function from NAME to the schema binding of balance and lost. This is isomorphic to the original state schema, World.

To complete these refactorings, and to preserve the full sense of the original specification, the original precondition and operation specifications have to be refactored to use the local and global states.

- The precondition schema declarations are not affected: purses are still identified by name, even though the name has been relocated to the global context.

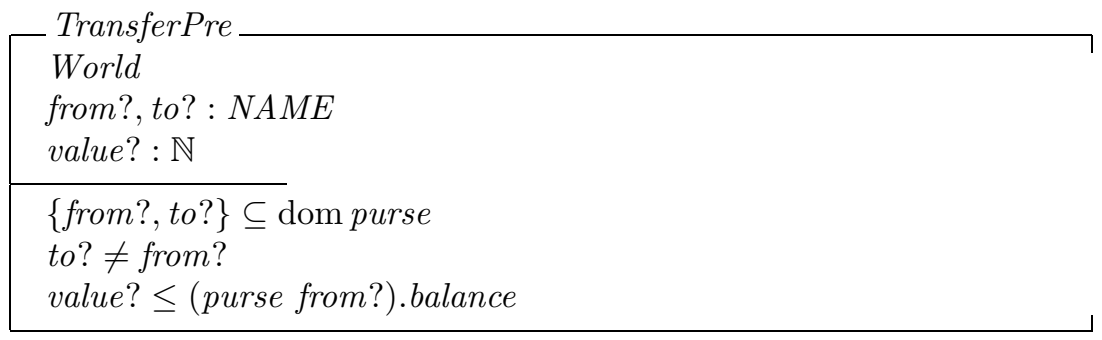

- Each reference to the balance function in the original precondition is replaced by a suitable reference to the purse function. Otherwise, the precondition predicates are unchanged.

The operations introduce global and local state schemas. To allow global operations to use the local state, explicit after-state purses are constructed. 
- A successful transfer involves explicitly two purses. The refactoring is influenced by the form of the elaboration pattern, multi-promotion, in its version for a fixed number of local instances. The declarations are made accordingly. Making a schema binding: ZRM [Stepney et al. 2003b] is used to construct purse instances.

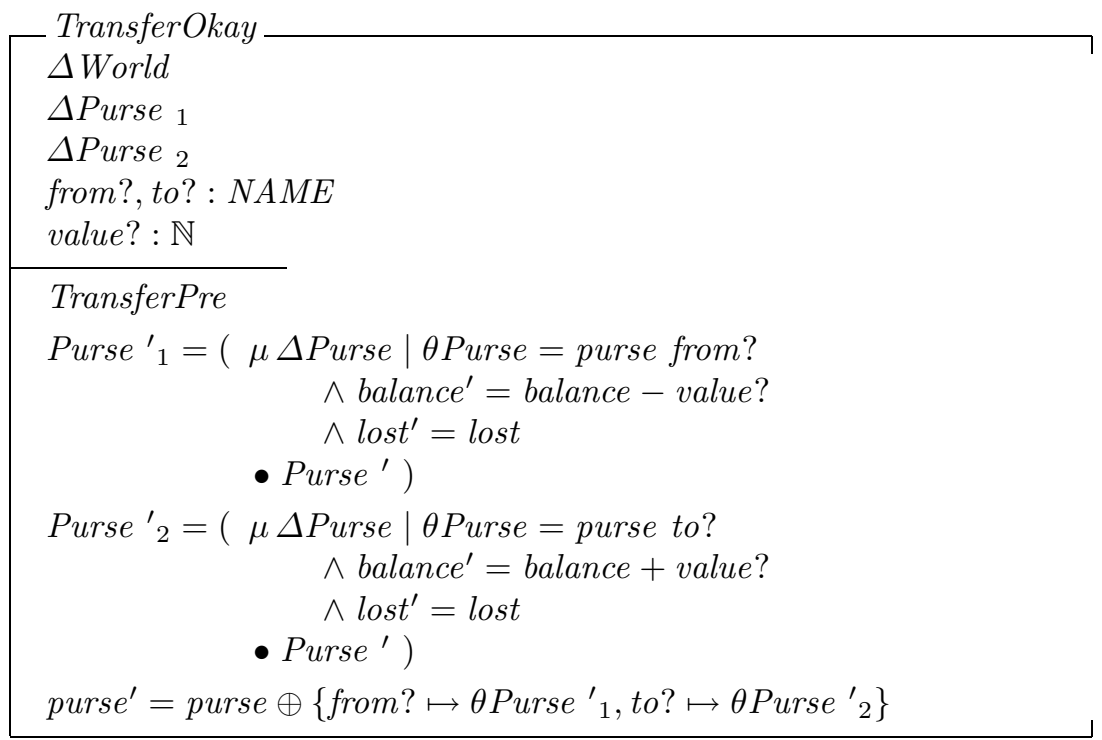

- Purse 1 has value? subtracted from its balance; no other elements is changed.

- Purse ${ }_{2}$ has value? added to its balance; no other elements is changed.

- The global world mappings to the instances of the two purses are overwritten with the after-states of each purse.

The lost case is refactored similarly.

- an unsuccessful transfer involves exactly one purse (we call this Purse ${ }_{1}$ to use it with the general framing schema)

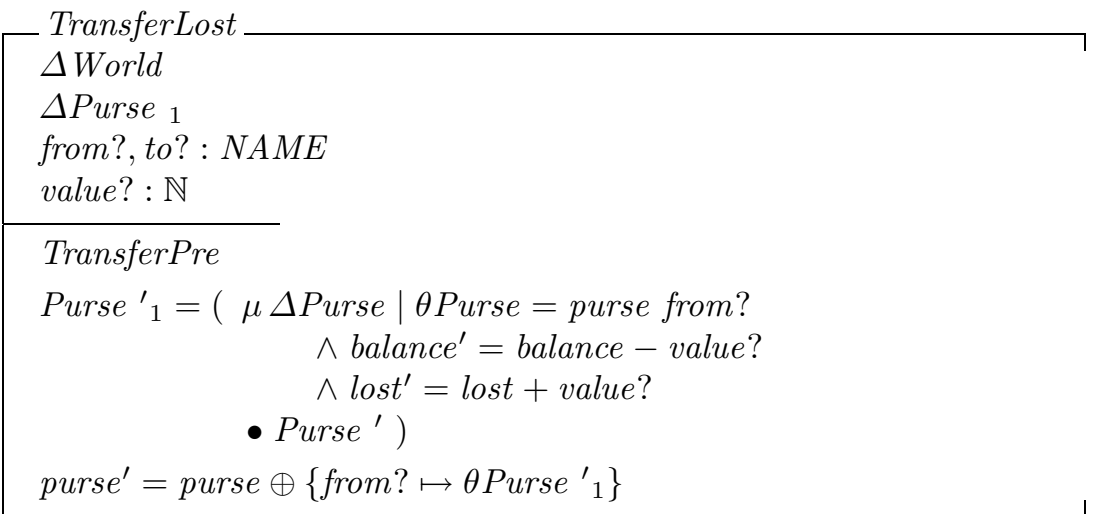


- Purse has value? subtracted from its balance, and added to lost

- the global world mapping to the purse is overwritten with the purse's afterstate

This intermediate refactoring preserves the meaning of the original specification, but is otherwise considerably more obscure than the original. Further refactoring is required ${ }^{1}$.

\subsection{Step 2: introduce local operations}

Having extracted the local and global states, attention now turns to the extraction of local operations. These are specified to include the local part of the precondition schema.

- the declaration of World has been replaced by the local Purse

- The from? and to? purse declarations are not relevant at the local level.

- The other declaration is as for the original precondition schema.

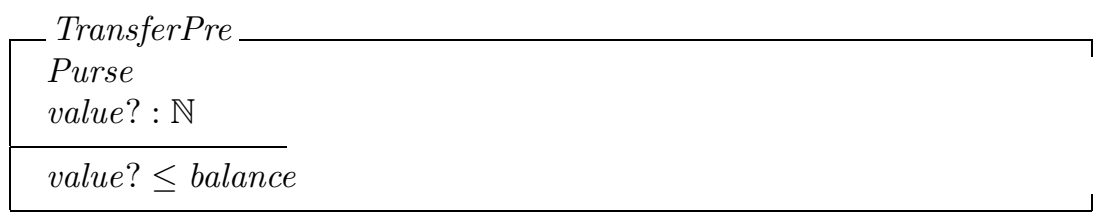

- The predicate is the remaining local one from the original precondition schema.

The local operations separately specify transfer from a purse, transfer to a purse and the lost transfer. The schemas are taken to be self-explanatory.

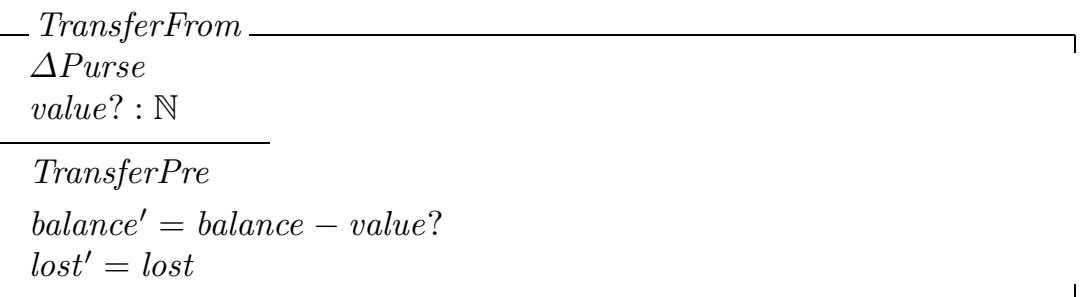

\footnotetext{
${ }^{1}$ This first refactoring is, in fact, closer to the form of the actual Purse specification [Stepney et al. 2000]. It was written in this form because the multi-promotion pattern was unknown to the authors at the time. See [Stepney et al. 2003b] for diagrams of the full specification.
} 
TransferTo

$\Delta$ Purse

value? : $\mathbb{N}$

TransferPre

balance $^{\prime}=$ balance + value?

lost' $=$ lost

TransferLost

$\Delta$ Purse

value? : $\mathbb{N}$

TransferPre

balance ${ }^{\prime}=$ balance - value?

lost ${ }^{\prime}=$ lost + value?

\subsection{Step 3: introduce framing schemas}

The next generative pattern is framing schemas. Each kind of operation requires a frame. All the local operations are simple changes to the local state. However, at the global level, a successful transfer updates the state of two purses whilst the unsuccessful transfer updates only one purse.

The validity of the purses involved in a transfer must be checked at the global level, since the name identifiers have been assigned only to the global state. This check needs to appear as part of the frame(s).

$\Phi$ Transfer

$\Delta$ World

$\Delta$ Purse $_{1}$

$\Delta$ Purse $_{2}$

from?, to? : NAME

value? : $\mathbb{N}$

$\{$ from?, to? $\subseteq$ dom balance

from? $\neq$ to?

$\theta$ Purse $_{1}=$ purse from?

$\theta$ Purse ${ }_{2}=$ purse to?

purse $^{\prime}=$ purse $\oplus\left\{\right.$ from? $\mapsto \theta$ Purse $^{\prime}{ }_{1}$, to $? \mapsto \theta$ Purse $\left._{2}{ }_{2}\right\}$

- the identities of the two purses are checked against the known identities, and are distinct 


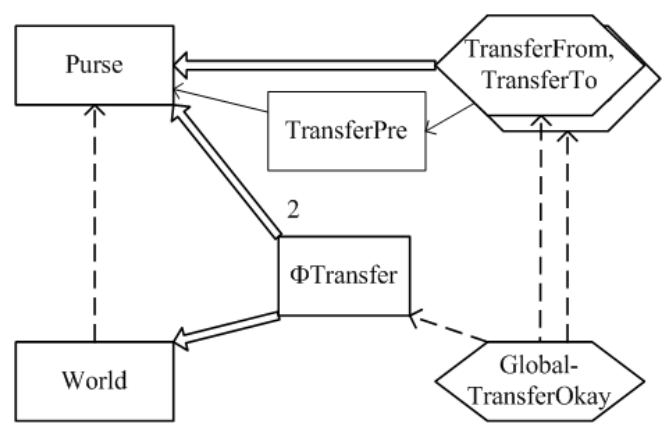

Fig. 3. Structure of the abstract world of electronic purses, after refactoring to a twostate promotion, for the GlobalTransferOkay operation

- the purse after-state is formed by overriding the mappings for the two instances with the results of the local operations

The single purse TransferLost could be provided with a framing schema by an application of the framing schemas pattern. However, it is equally valid, and requires fewer definitions, to think of the unsuccessful global operation as affecting two purses, one of which is unchanged.

\subsection{Step 4: Define the global operations}

The final step applies the global operations pattern.

$$
\begin{aligned}
& \text { GlobalTransferOkay }==\exists \Delta \text { Purse }_{1} ; \Delta \text { Purse }_{2} \bullet \\
& \Phi \text { Transfer } \wedge \text { TransferFrom }{ }_{1} \\
& \wedge \text { TransferTo }{ }_{2}\left[\text { value }{ }_{1} / \text { value }_{2}{ }_{2}\right] \\
& \text { GlobalTransferLost }==\exists \Delta \text { Purse }_{1} ; \Delta \text { Purse }_{2} \bullet \\
& \text { TTransfer } \wedge \text { TransferLost }{ }_{1} \wedge \Xi \text { Purse }_{2}
\end{aligned}
$$

\subsection{Resulting specification, summary}

Gathering together all the pieces produced above, the complete specification of the abstract purse world comprises (a) local specifications, (b) framing schema(s) and (c) global transfer operations that promote the local transfer elements. This could be demonstrated to be isomorphic to the original by a schema expansion refactoring [Stepney et al. 2002] of both versions, and simplifying. Alternatively, conjectures on the operations could be formulated and proved. Neither is demonstrated here.

The specification using promotion is slightly longer, but more readable. Writing further operations that change purses is straightforward: the local component operations are added, and the global operation pattern applied.

Figure 3 represents the structure of the specification after refactoring. This diagram looks more complicated than the pre-refactoring diagram (figure 2); this 
demonstrates how much specification structure was implicit before (hidden in the state and operation schemas). More explicit structure makes a specification easier to comprehend.

\section{Conclusion}

The refactorings shown here take an unstructured specification into a specific pattern by a series of meaning preserving refactoring steps, guided by the target patterns and sub-patterns.

Refactoring can also be used to take a specification in one pattern in to a different pattern: $\mathrm{Z}$ patterns include a number of choice patterns that are equivalent (in some sense) formulations of the same concept; some are better for communication, and some are better for proof or refinement.

Meaning preservation is a crucial component in refactoring. Whilst it may be desirable to evolve the meaning of a specification by a series of small, controlled steps, this is not what is normally meant by refactoring. (In [Stepney et al. 2002] we refer to this as benefactoring.) In code refactoring, the meaning preservation is demonstrated if all the original functionality is preserved by the refactoring (same inputs lead to same outputs). In specification, meaning preservation may be defined in terms of isomorphic semantic models; it may be defined in terms of mutual refinement; it may be defined in terms of what (refactored) conjectures can be proved true (and, if relevant, proved false). France (private communication) uses a much looser definition of meaning preservation, relating to the intent of the specifiers, a preservation that could not be mechanically checked. The definition can be chosen to suit the application.

Some refactoring is clearly supportable by tools: some tools can already refactor, as part of a proof-assistant role. Thus, schemas can be expanded, preconditions calculated, and other equivalent structures substituted. These refactorings follow a strict mathematical meaning preservation. However, practical refactoring is more likely to need to preserve the properties of a specification, and to demonstrate preservation by reproving the conjectures. This occurred during the commercial specifications that are the motivation for this paper [Cooper \& Stepney 2000], [Stepney \& Cooper 2000].

More work on refactoring proofs is under way. Such refactorings, especially where guided by patterns, have the potential to guide proof, refinement, and other aspects of formal software engineering.

\section{Acknowledgements}

We thank the anonymous referee whose careful reading of an earlier version of this paper resulted in many improvements.

\section{References}

[Alexander et al. 1977]

Christopher Alexander, Sara Ishikawa, Murray Silverstein, Max Jacobson, Ingrid 
Fiksdahl-King, and Shlomo Angel. A Pattern Language: Towns, Buildings, Construction. Oxford University Press, 1977.

[Barden et al. 1994]

Rosalind Barden, Susan Stepney, and David Cooper. Z in Practice. BCS Practitioner Series. Prentice Hall, 1994.

[Bowen et al. 2000]

Jonathan P. Bowen, Steve Dunne, Andy Galloway, and Steve King, editors. ZB2000: First International Conference of B and Z Users, volume 1878 of LNCS. Springer, 2000.

[Cooper \& Stepney 2000]

David Cooper and Susan Stepney. Segregation with communication. In [Bowen et al. 2000], pages 451-470.

[d'Inverno \& Luck 2001]

Mark d'Inverno and Michael Luck. Understanding Agent Systems. Springer, 2001.

[Fowler 1999]

Martin Fowler. Refactoring: improving the design of existing code. Addison-Wesley, 1999.

[Gamma et al. 1995]

Erich Gamma, Richard Helm, Ralph Johnson, and John Vlissides. Design Patterns. Addison-Wesley, 1995.

[Morgan \& Sufrin 1984]

Carroll Morgan and Bernard Sufrin. Specification of the UNIX filing system. IEEE Trans. Softw. Eng, 10(2):128-142, 1984.

[Stepney \& Cooper 2000]

Susan Stepney and David Cooper. Formal methods for industrial products. In [Bowen et al. 2000], pages 374-393.

[Stepney et al. 1992]

Susan Stepney, Rosalind Barden, and David Cooper, editors. Object Orientation in Z. Springer, 1992.

[Stepney et al. 2000]

Susan Stepney, David Cooper, and Jim Woodcock. An electronic purse: Specification, refinement, and proof. Technical Monograph PRG-126, Programming Research Group, Oxford University Computing Laboratory, 2000.

[Stepney et al. 2002]

Susan Stepney, Fiona Polack, and Ian Toyn. Refactoring in maintenance and development of $\mathrm{Z}$ specifications and proofs. In REFINE 2002, Copenhagen, volume 70(3) of ENTCS. Elsevier, 2002.

[Stepney et al. 2003a]

Susan Stepney, Fiona Polack, and Ian Toyn. An outline pattern language for Z. 2003. (these proceedings).

[Stepney et al. 2003b]

Susan Stepney, Fiona Polack, and Ian Toyn. A Z patterns catalogue I: specification and refactoring, v0.1. Technical Report YCS-2003-349, York, 2003.

[Woodcock \& Davies 1996]

J. C. P. Woodcock and J. Davies. Using Z: Specification, Refinement, and Proof. Prentice-Hall, 1996. 